\title{
Test of the Accuracy of Approximate Methods to Handle Distant Binary-Single Star Encounters
}

\author{
Yoko Funato $^{1}$, D.C. Heggie ${ }^{2}$, P. Hut ${ }^{3}$ and Jun Makino ${ }^{4}$ \\ ${ }^{1}$ Department of Arts and Sciences, University of Tokyo, \\ Present address: General System Studies, Dept. Arts and Sciences, University of Tokyo, \\ Komaba, Meguro-ku, Tokyo, 153 Japan \\ email: funato@artcompsci.org \\ ${ }^{2}$ School of Mathematics, University of Edinburgh, UK \\ email:d.c.heggie@ed.ac.uk \\ ${ }^{3}$ Institute for Advanced Study, USA \\ email:piet@ias.edu \\ ${ }^{4}$ Division of Theoretical Astronomy, National Astronomical Observatory Japan, \\ email:makino@cfca.jp
}

\begin{abstract}
In the numerical simulations of evolution of star clusters, binary-single star interactions frequently take place. Since the direct integration of them is time consuming, distant interactions between binaries and field stars are often integrated by using some approximations. Traditionally the effect of the error caused by the approximated treatment is regarded as small enough to be ignored. However, if we have a binary-dominated core, the energy drift is large.

In this study, we perform numerical experiments to evaluate the effect of neglecting the weak perturbation from distant single particles. We developed an $N$-body integrator which can manipulate multiple precision floating point numbers.
\end{abstract}

Keywords. methods:n-body simulations

\section{Introduction}

\subsection{A Binary and a single star interactions}

Binary-single star interactions have an important role for the evolution of star clusters. Though the importance of binary-single star interactions has been well established, to evaluate their effect quantitatively is difficult and requires numerical simulations. However, the numerical simulation of the binary-single star interactions in star cluster evolution is also difficult even for the cases of weak interactions, such as distant encounters of binary-single stars and quasi-stable hierarchical triples. In this study, we investigate the correctness of the approximated techniques used in the numerical study of these weak interactions.

In order to numerically integrate accurately, the stepsize should be less than 0.01 times the period of the binary. The stepsize is much smaller than the orbital time of the encounter so that the number of steps becomes large. This causes two problems: too long computing and growth of accumulation of round-off errors.

To avoid these problems, usually approximated techniques are employed. One of which is an individual time step algorithm. The other is a single star approximation of the binary.

Though these techniques have been used, the adequacy of these approximations is not well understood. There are several mechanisms which may not be treated correctly in 

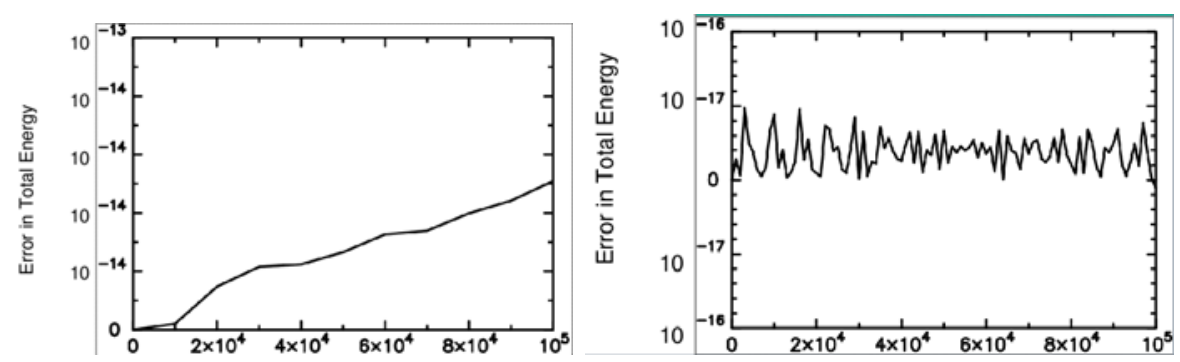

Figure 1. Growth of errors in total energy. Left: double precision Right: octuple precision

previous numerical studies. First, it is not well known when a binary can be treated as a single mass and when it should be treated as two stars. Second, though the energy can be regarded as an adiabatic invariant, the evolution of eccentricity of inner binary is not negligible (e.g., Heggie \& Rasio, 1996). Third, the interactions between stars with large different time scale, such as stars in the core and those in the halo, are also important for the entire cluster evolution (Makino 2007; Nitadori 2007).

We plan to do numerical experiments to check the correctness of the approximate treatments of binaries. In order to avoid the accumulation of round-off errors, a high precision integrator is required. Using this integrator, we will carry out numerical experiments to estimate effect of weak distant encounters to cluster evolution.

\section{Numerical experiment}

For our experiment, we wrote an integrator which can manipulate any precision floating number. In our integrator, the precision is a free parameter. For the integrator we implemented the time-symmetric 4th order Hermite scheme to avoid an error growth due to truncation errors.

In Fig. 1 the result of the integration of the evolution of a hierarchical triple is shown. In Fig. 1, the left and right panels correspond to a double and octuple precision calculation, respectively. In this calculation the stepsize is set so as to the truncation error per one step is $\sim 10^{-16}$. Here the errors in total energy is plotted against time. In the left panel, the growth of error takes place. This growth is due to the accumulation of round-off errors. The right panel shows the result using the octuple precision integrator. Contrary to the result shown on the left panel, no error growth is observed. These figures show that our integrator works as well as expected.

Using this integrator, we are going to perform experiments of binary-single star interactions in future.

\section{References}

Heggie, D. C. \& Rasio, F. 1996, MNRAS. 282, 1064

Makino, J. 2007, this volume

Nitadori, K. 2007, this volume 